\title{
RSSDI consensus on self-monitoring of blood glucose in types 1 and 2 diabetes mellitus in India
}

\author{
P. V. Rao ${ }^{1}$ • B. M. Makkar ${ }^{2} \cdot$ Ajay Kumar $^{3}$ - A. K. Das ${ }^{4} \cdot$ A. K. Singh ${ }^{5} \cdot$ Ambrish Mithal $^{6} \cdot$ Anil Bhansali $^{7} \cdot$ Anoop Misra $^{8,9}$ • \\ Anuj Maheshwari ${ }^{10}$. Arvind Gupta $^{11}$ - Ashu Rustogi ${ }^{12}$ • Banshi Saboo ${ }^{13}$. C. H. Vasanth Kumar ${ }^{14}$. \\ C. R. Anand Moses ${ }^{15} \cdot$ Hemant Thacker ${ }^{16}$. Jayant Panda ${ }^{17}$. Jayaprakashsai Jana ${ }^{14} \cdot$ Jothydev Kesavdev $^{18}$. \\ K. R. Narasimha Setty ${ }^{19}$. Manoj Chawla ${ }^{20}$ • Neeta Deshpande ${ }^{21} \cdot$ Nikhil Tandon $^{22} \cdot$ Rajeev Chawla ${ }^{23} \cdot$ Rajeev Kovil $^{24}$. \\ Rakesh Sahay ${ }^{25}$. S. V. Madhu ${ }^{26}$. Samar Banerjee ${ }^{27}$. Sanjay Agarwal ${ }^{28}$. Sanjay Kalra ${ }^{29} \cdot$ Sarita Bajaj $^{30}$. \\ Shashank R Joshi ${ }^{31}$. S. R. Aravind ${ }^{32}$ • Subhankar Chowdhury ${ }^{33}$. Sujoy Ghosh ${ }^{34}$ - Sunil Gupta ${ }^{35}$. \\ Viswanathan Mohan ${ }^{36}$. Vijay Panikar ${ }^{37}$. Vijay Viswanathan ${ }^{38}$
}

Published online: 28 August 2018

(C) The Author(s) 2018

\begin{abstract}
Maintaining a good glycemic control is crucial in the management of diabetes mellitus (DM) as it is associated with the reduction in both macro and microvascular complications of the disease. Self-monitoring of blood glucose (SMBG), which provides the day-to-day blood glucose levels, is a simple and practical tool for maintaining a good glycemic control. Although SMBG is widely practiced in other countries, its use in India is very limited. Even when used, it is not carried out is a structured manner. There seems to be a lack of education about the purpose of SMBG and the correct process and schedule to be followed. This highlights the unmet need for country-specific SMBG recommendations. In order to fulfil this need, a panel of expert endocrinologists/ diabetologists came together under the aegis of Research Society for the Study of Diabetes in India (RSSDI). They reviewed the current literature, combined the evidences with their clinical knowledge and expertise, and developed consensus recommendations for SMBG practice in India. This document provides a comprehensive review of the current literature on SMBG and presents the recommendations made by the expert panel.
\end{abstract}

Keywords Diabetes Mellitus · Glucose Meters · Glycemic Control · SMBG · Self-monitoring of blood glucose $\cdot$ Type 1 DM . Type 2 DM

\section{Introduction}

Diabetes mellitus (DM) is a chronic illness that needs longterm multidisciplinary care. It accounts for a significant burden due to the associated morbidity, mortality, and healthcare resource utilization [1, 2]. Management is primarily targeted towards prevention of acute and chronic complications, for which constant efforts are being made to test novel interventions to improve outcomes [3]. Patient awareness and active participation in self-care to prevent both acute and long-term

B. M. Makkar

drbmmakkar@gmail.com

Extended author information available on the last page of the article complications are equally important for effective management of this disorder [4].

As per the International Diabetes Federation (IDF), about 425 million people are affected with DM worldwide, and this number is estimated to reach 629 million by the year 2045 . India ranks second in the world, closely following China, with almost 73 million Indians living with diabetes. With a projected prevalence of over 134 million, India is estimated to surpass China by the year 2045 [5].

Rapid increase of diabetes burden in India seems to be due to a combination of various factors including genetic predisposition, urbanization, and lifestyle changes such as sedentary lifestyle and changing nutritional habits [6-8]. Thus, diabetes is a major public health concern in India. On the brighter side, with the development of science and technology, newer methods to diagnose, monitor, and treat DM have enabled management of this condition more effectively. Nevertheless, several patients 
still struggle to reach therapeutic targets and are, therefore, at an increased risk of developing complications. Long-term complications of diabetes are well known to occur, especially in patients with poor glycemic control. Hyperglycemia associated with diabetes leads to both macro- and microvascular complications. Macrovascular complications include coronary artery disease leading to angina and/or myocardial infarction and peripheral artery disease that may lead to stroke, diabetic encephalopathy, and diabetic foot [9]. Microvascular complications include nephropathy, neuropathy, and retinopathy. Unarguably, these micro- and macrovascular complications of diabetes are the cause of real burden of the disease [5]. In addition, it has been found that the cost of treatment of patients with complications is much higher than that of patients without complications [10-12]. It is, therefore, essential to put all the efforts towards preventing these complications.

Glycemic level is known to be directly associated with vascular complications of diabetes [13-15]. Moreover, there is strong evidence that good glycemic control is associated with the reduction in both macro- and microvascular complications [16-20]. Thus, maintaining a good glycemic control is of utmost importance for adequate management of diabetes. Glycated hemoglobin (HbAlc), which denotes the average level of blood glucose over about 3 months, and self- monitoring of blood glucose (SMBG), which provides the day-to-day blood glucose levels, are two important tools for monitoring of glycemic control. Fructosamine test is another tool, which denotes the blood glucose levels over the past 2 to 3 weeks. Another such tool is continuous glucose monitoring (CGM), which measures interstitial fluid glucose levels continuously for varying duration of time [21-24].

Evidence suggests that the glycemic variability or extreme changes in blood glucose (hypoglycemia or hyperglycemia) levels could have a role to play in the development of longterm complications independent of $\mathrm{HbAl} \mathrm{c}$ levels, and the risk of these complications could be reduced by better daily control of blood glucose [25]. A recent study (DEVOTE 2) found that higher day-to-day fasting glycemic variability is associated with increased risks of severe hypoglycemia and all-cause mortality [26]. Evidence also indicates that blood glucose variability can have several other effects including increased cardiovascular and cerebrovascular risk, increased risk of cognitive impairment in elderly patients, and deterioration of endothelial and renal dysfunction [27-30]. All these evidences further highlight the importance of a tool that can assess the glycemic variability on a daily basis. SMBG is the simplest and possibly most practical tool to assess the effectiveness and safety of glycemic control and will be reviewed here.

Disease burden of DM in India is increasing. Long-term complications, which form the main burden of disease, can be reduced by maintaining a good glycemic control.

\section{What is SMBG?}

SMBG refers to testing and recording of blood glucose levels by a patient and/or caretaker, at home or in hospital, at different times of the day [21, 31, 32]. The blood glucose levels obtained help patients and clinicians to make appropriate adjustments in lifestyle (diet and physical exercise) and medications [31].

\section{SMBG technique}

Before performing SMBG, hands should be washed with soap and water and dried thoroughly. The glucose meter should be prepared. Preparation may vary slightly depending on the glucose meter brand and, therefore, it is important to read the user manual carefully before using the glucose meter. A test strip should be inserted into the glucose meter. A lancet/pricking device should be used to prick the finger. It is advisable to alternate between fingers as they tend to become sensitive over time. After pricking, if required, the finger can be gently massaged in the direction of the prick to help form a drop of blood. The drop of blood should be placed on the correct spot on the test strip as indicated in the user manual. The glucose meter will display the glucose reading within a few seconds. In most glucose meters, the units can be changed from millimoles per liter to milligrams per deciliter and vice versa. Most glucose meters store the results for weeks and can be retrieved later. These readings will enable the patient/clinician to make lifestyle/therapeutic adjustments. Used test strip and lancets should be disposed of properly as per recommendations to avoid contamination. Test strips and glucose meter should be kept away from sunlight and should also be protected from moisture. Most of the manufacturers recommend that once a bottle of test strips is opened, they should be used within 90 days of opening or the expiry date mentioned on the bottle, whichever is earlier. Some of the common sources of errors to be considered for SMBG are listed in Table 1 [33].

\section{Structured SMBG}

It is important to understand that just recording blood glucose levels on a daily basis is not enough, if not acted upon. In order to be clinically relevant and implemented successfully, SMBG must be conducted in a structured way. Structured SMBG 
Table 1 Common sources of errors while conducting SMBG

\begin{tabular}{ll}
\hline Problem/error & Advice/recommendation \\
\hline Test strip not fully inserted into glucose meter & $\begin{array}{l}\text { Remove the test strip and reinsert it. Always ensure that the } \\
\text { test strip is fully inserted in the glucose meter } \\
\text { Discard the test strip and repeat the test }\end{array}$ \\
$\begin{array}{l}\text { Not enough blood was drawn into the test strip } \\
\text { for measurement }\end{array}$ & $\begin{array}{l}\text { Always clean and dry the site before sampling } \\
\text { fingertip is contaminated with sugar }\end{array}$ \\
$\begin{array}{l}\text { Not enough blood applied to strip } \\
\text { Batteries low on power }\end{array}$ & $\begin{array}{l}\text { Repeat test with a new strip } \\
\text { Change batteries and repeat the test }\end{array}$ \\
& $\begin{array}{l}\text { Results from alternative sites may not match fingerstick results } \\
\text { Site validated by the manufacturer must be used }\end{array}$ \\
\hline
\end{tabular}

(sSMBG) involves checking the blood glucose levels at predefined times each day [32]. It is a methodical approach to blood glucose monitoring, which enables the patients and clinicians to understand the blood glucose pattern throughout the day, so that appropriate therapeutic adjustments can be made. Along with the blood glucose levels, patients must also record their food intake and physical activity. sSMBG also involves imparting proper education and motivation to the patients and proceeding only after judging their willingness. Education should not focus just on how to conduct SMBG and how to adjust the medication based on the individual readings but should also include explaining to the patients the importance of good control [34]. The physician's role is to regularly review the SMBG data at every follow-up visit, and to discuss the SMBG readings with the patient. Patients can be advised to make minor adjustments of insulin dosage and to incorporate appropriate lifestyle changes based on SMBG readings. The clinician himself must have proper knowledge, training, and experience to closely follow the blood glucose readings, and understand the pattern to be able to prescribe appropriate changes to diet, exercise, and/or medications. Patients must be educated about the target glucose levels as per guidelines and their importance. Patients and clinicians must agree on the target levels of blood glucose and also on the timing and frequency of testing. At each stage, proper feedback must be given to the patients including an explanation of the potential causes of low or high blood glucose levels. The action plan for maintaining blood glucose levels within target range must be also explained to the patient in a clear manner and it must be agreed upon mutually $[32,35]$.

In short, sSMBG occurs when the clinician and the patient both express their willingness and are motivated to perform the entire process, possess knowledge to interpret the glucose levels correctly, understand the pattern, and take appropriate actions towards achieving a good glycemic control [32, 35].

Benefits of structured over unstructured SMBG are well documented [35-42]. Also, evidence suggests that lack of knowledge about how to interpret the results of SMBG and how to adjust the dose based on those results is the main deterrent in the success of SMBG, further emphasizing the importance of sSMBG. It has been demonstrated that SMBG is of limited value when it is not applied in a structured fashion [43].

SMBG is an important tool for monitoring blood glucose levels. SMBG should be structured for it to be effective.

\section{What are the advantages of SMBG?}

SMBG plays a very important role in monitoring the plasma glucose levels on a day-to-day basis. SMBG complements $\mathrm{HbA} 1 \mathrm{c}$ testing in evaluation and monitoring of glycemic control. While HbA1c reflects the glycemic status over weeks, SMBG provides day-to-day fluctuations in blood glucose levels. Measurement of 2-h glucose level, which can be obtained with SMBG, is considered to be a stronger predictor of cardiovascular disease as compared to HbAlc. Also, in some conditions such as hemoglobinopathies, malaria, anemia, and blood loss, HbA1c level for glycemic control may not be reliable, and SMBG plays a major role here [44]. Moreover, in pregnancy, greater emphasis is placed on SMBG than on HbAlc [45].

SMBG is crucial in the management of insulin-treated patients, and its role in patients on non-insulin treatment has also been recognized [36, 37, 39, 40, 42, 46-48]. SMBG enables patients to detect acute hypoglycemia/hyperglycemia and take appropriate action in coordination with their clinicians [49]. Thus, it plays a vital role in ensuring safety of patients, especially those on intensive insulin therapies. It also helps patients feel more in control and more empowered in the management of their diabetes. They learn how their behavior, in terms of diet or physical exercise, may affect their blood glucose levels, and feel encouraged to act more responsibly and take 
informed decisions related to their health. Patients can see positive effects of modifying their diet and exercise in real time, which further drives them to continue their efforts.
Thus, in addition to controlling their blood glucose levels, sSMBG also helps weight management in these patients [50].

SMBG helps in maintaining a good glycemic control by generating data for therapeutic and lifestyle adjustments. It detects acute hypoglycemia/hyperglycemia and protects patients against extreme glucose variations.

\section{What are the challenges associated with SMBG and how to overcome them?}

While SMBG has several advantages, there are also some challenges associated with it. SMBG is a procedure that requires active participation by the patients. Patients may find SMBG inconvenient, painful, and cumbersome [51]. They may find it difficult to integrate SMBG in their daily routine [52]. Another hurdle is ignorance of patients towards the seriousness of diabetes and its complications. Cost of the test strips and needles is another concern especially for patients who have to pay for their healthcare themselves. Carrying the glucose meter with them while traveling is another barrier [51]. Undesired readings on glucose meter may also discourage patients from wanting to continue SMBG. Patients may feel that SMBG affects their quality of life [53]. Additionally, depression has also been documented in patients performing SMBG [54]. Another challenge is the unavailability of diabetes care team for titration of the doses and providing appropriate guidance to the patients.
Most of these barriers or challenges associated with SMBG can be overcome by proper communication between the patients and their clinicians/diabetes care providers. Patients may disregard the seriousness of long-term complications and therefore may display low motivation for treatment. The effects of uncontrolled blood glucose levels and day-to-day glycemic variability on long-term health should be properly explained to the patients. Patient beliefs and values must also be considered. It is of utmost importance that clinicians take sufficient time to explain the importance of SMBG to their patients so that they understand the rationale for SMBG and are encouraged to follow the instructions for conducting and recording blood glucose readings as advised. Initially, attainable targets should be set, which will give the patients a sense of achievement, and motivate them further to continue SMBG. Also, therapeutic targets recommended by guidelines should be explained to the patients and must be agreed upon by both clinicians and patients as this has been shown to improve patient outcomes [55].

Challenges of SMBG can be overcome by a proper communication between the clinician and the patient and by ensuring that SMBG is carried out in a structured manner.

\section{Importance of accuracy of SMBG systems}

Accuracy of SMBG systems is very important for the results to be reliable and safe. It has a direct effect on therapeutic decisions and may also have long-term implications. SMBG systems should comply with the International Organization for Standardization (ISO) 15197: 2013 requirements [56]. Freckmann et al. conducted a study to examine the different SMBG systems and found that 7 of the 34 systems evaluated did not fulfill the minimal accuracy requirements of ISO. Regular evaluation of the blood glucose meters is, therefore, of utmost importance [57].

SMBG devices have been associated with a number of user errors such as using expired test strips, inadequate storage conditions, or glucose-contaminated fingertips that compromise the analytical performance. In order to reduce potential user errors, more integrated systems (incorporation of the tests into the meter by using cassettes, discs, or drums) have been developed. Baumstark et al. carried out a study to evaluate the system accuracy of this improved system based on ISO 15197:2013, clause 6.3, for three reagent system lots. The study reported a high level of accuracy; $100 \%$ within the defined limits in the hands of trained study personnel and $99.1 \%$ in the hands of intended users [58].

Another technical challenge is that there is a difference between glucose levels in the venous and capillary blood with venous blood having a lower concentration of glucose. The difference varies between fasting and post-meal. The difference is not much at fasting but there is a larger difference after a meal [59]. The revised ISO 15197: 2013 requirements specify tighter accuracy standards (when compared with ISO 15197: 2003) requiring that $95 \%$ of blood glucose results should reach the following standard:

- Within $\pm 15 \mathrm{mg} / \mathrm{dL}$ of laboratory results at concentrations $<100 \mathrm{mg} / \mathrm{dL}$ 
- Within $\pm 15 \%$ of laboratory results at concentrations $\geq$ $100 \mathrm{mg} / \mathrm{dL}$

The 2013 guidelines also specify that $99 \%$ of the individual glucose results must fall within zones $\mathrm{A}$ and $\mathrm{B}$ of the Consensus Error Grid for type 1 DM [56]. Some glucose meters currently available in our country which conform to ISO 15197:2013 standards include Accu-Chek Performa, GluNEO Lite, Contour TS, One Touch Verio Flex, Alere G1, and SD Check Gold.

One more challenging aspect is the commonly used graphs and plots to assess the accuracy of SMBG systems, which get increasingly difficult to comprehend as the number of data points increase. Recently, a new approach of displaying SMBG measurement accuracy data has been introduced called the "rectangular target plot" (RTP), which presents data in a simple yet comprehensible manner [60]. RTP was evaluated by creating plots for 50 SMBG systems and 87 reagent system lots from 8 manufacturers. It was found that RTP remained comprehensible even when data was displayed from multiple reagent system lots or products and was completely applicable in more than $93 \%$ of the cases analyzed [61].

Also, it is important to ensure that validation and calibration of the device is carried out properly.

SMBG systems compliant with ISO 15197:2013 should be used to ensure that the results obtained are reliable.

\section{What is the evidence of effectiveness of SMBG?}

SMBG is commonly used in developed nations as an integral part of diabetes management [62]. In a survey conducted in Canada in 2011, almost $90 \%$ of the patients with type $2 \mathrm{DM}$ reported using SMBG. Further, there was no significant difference between patients using insulin only and those taking insulin plus oral medication or an oral medication only although frequency of SMBG was lower in these patients [62]. In another survey conducted in the UK, $80 \%$ of the 554 respondents reported high satisfaction with SMBG. They also reported that SMBG helped them feel more "in control" of their diabetes management [63].

Several studies have demonstrated that SMBG helps in better glycemic control and is thus essential in the management of DM [36, 40, 47, 64-70].

\section{In type 1 DM patients}

Patients with type 1 DM experience higher glucose variability leading to a greater risk of hypoglycemia. Therefore, SMBG plays a critical role in the management of these patients. The landmark Diabetes Control and Complications Trial, which was the first long-term randomized study including 1441 patients with type $1 \mathrm{DM}$, showed that intensive therapy guided by frequent blood glucose monitoring when compared with conventional therapy (with one or two daily insulin injections) was associated with delayed onset and slowed progression of microvascular complications [71]. The results of this study were published in 1993 and since then, use of SMBG gradually increased, and it is now routinely practiced in patients with type $1 \mathrm{DM}$. It has also been found that higher frequency SMBG in these patients is strongly associated with lower HbA1c levels [67, 70]. Thus, SMBG is absolutely essential for achieving and maintaining optimal blood glucose levels in all patients with type $1 \mathrm{DM}$ including children, adolescents, and adults.

\section{In type 2 DM patients on insulin therapy}

As in patients with type $1 \mathrm{DM}$, there is no doubt that SMBG has a very important role to play in the management of patients with type $2 \mathrm{DM}$ who are on insulin therapy. SMBG has been universally recognized as an integral part of insulin regimens. SMBG not only adds value but is crucial in patients especially on the complex insulin regimens. It ensures safety and efficacy of the insulin regimens [41, 42, 72-74].

\section{In type 2 DM patients on non-insulin therapy}

Evidence for the utility of SMBG in patients who are not on insulin therapy has been equivocal [75]. While some evidence suggests that SMBG may help in reduction of HbA1c in this group of patients, other studies have found that the advantage of SMBG in these patients is only modest, if at all [76]. A review of six randomized controlled trials (RCTs), showed that patients with type 2 diabetes on non-insulin treatment had a statistically and clinically relevant reduction of $\mathrm{HbA1c}$ by $0.39 \%$ with SMBG when compared with the control groups [77]. On the other hand, a meta-analysis found SMBG in type 2 patients of non-insulin therapy to be only modestly effective in reducing HbA1c [78].

Two systematic reviews, published in the year 2012, concluded that there is only limited benefit with SMBG in type 2 non-insulin-treated patients [79, 80]. The authors of one of these studies, which was a Cochrane review including 12 RCTs $(N=3259)$, concluded that the overall effect of SMBG in patients on non-insulin treatment was only small at short term and decreased after a duration of 1 year [79]. It is 
important to note that the credibility of this Cochrane review has been questioned [81]. In the other study, which was a meta-analysis including six RCTs $(N=2552)$, although there was a statistically significant difference in the level of $\mathrm{HbAlc}$ between the groups with or without SMBG, the authors concluded that individual patient data was not convincing for a clinically meaningful effect [80].

On the other hand, some individual studies have found SMBG to be useful even for patients on non-insulin therapy. In a long-term epidemiological cohort study, 3268 patients with type 2 diabetes were followed for a mean duration of 6.5 years [47]. SMBG was associated with decreased diabetes-related morbidity and all-cause mortality in overall study population and also in a subgroup of patients who were not receiving insulin therapy. In the subgroup on non-insulin therapy, SMBG was associated with a reduced risk of non-fatal ( $\mathrm{HR}=0.60,95 \%$ CI 0.44 $0.82 ; p<0.001)$ and fatal endpoints $(\mathrm{HR}=0.54,95 \% \mathrm{CI}$ $0.33-0.87 ; p=0.010)$ [47].

Experts believe that when patients, especially those on noninsulin therapy, do not benefit from SMBG, it is mainly because the process is not conducted in a structured format. The Structured Testing Program (STeP) study was a 12-month study that compared outcomes in patients receiving enhanced usual care with those receiving structured SMBG [36]. sSMBG was associated with a statistically significant reduction in $\mathrm{HbAlc}$ levels in both intention-to-treat analysis ($0.3 \% ; p=0.04)$ and per protocol analysis $(-0.5 \% ; p<$ 0.003) [36]. At the IDF 2017 congress, Parsons et al. presented the results of a 12-month multicenter RCT that assessed the efficacy of SSMBG in patients on non-insulin therapy with poor glycemic control $(\mathrm{HbA} 1 \mathrm{c} \geq 7.5 \% \leq 13 \%)$. They found that use of sSMBG provided clinically and statistically significant benefits with a mean reduction in HbAlc of $0.9 \%$ (95\% CI -1.18 to $-0.62 ; p=<0.001)$. Levels of satisfaction with SMBG remained high throughout the course of the study and only low levels of anxiety or pain caused by SMBG were reported [82].
There have been some reports of undesirable impact of SMBG on patients such as effects on quality of life (DiGEM study) and depression (ESMON study) [53, 54]. However, this is thought to occur when the physician is not involved enough in the care and when patients are not well-educated about the procedure of SMBG [83]. This further emphasizes the importance of SSMBG. Proper education of patients is very important including the action to be taken when blood glucose levels are out of the target range [72, 73]. Additionally, some studies have found that SMBG when conducted correctly can, in fact, reduce the stress and depression associated with diabetes. A 12-month cluster-randomized trial $(N=483)$ was conducted on non-insulin-treated type 2 patients specifically to assess whether SSMBG reduces depressive symptoms and diabetes distress. Patients were divided into experimental (structured SMBG) and active control groups. Although both groups had significant improvement in depression and disease-related distress $(p<0.01$ in both groups), experimental patients displayed significantly greater reductions in distress related to regimen adherence than controls. Further, those experimental patients who had elevated diabetes distress or depressive symptoms at baseline showed significantly greater reductions in distress and depressive symptoms than control patients at 12 months [84]. In another study SSMBG was associated with significant increases in self-confidence and autonomous motivation associated with diabetes self-management [39].

Several other studies have demonstrated clear benefit of SMBG in the management of patients with non-insulintreated type 2 DM [50, 65, 66, 69, 85-89]. Shiraiwa et al. demonstrated that lesser frequency of SMBG (10 times per month) in addition to being cost-saving was also effective in improving glycemic control. The mean decrease in $\mathrm{HbA} 1 \mathrm{c}$ was significantly more $(p=0.028)$ in the SMBG group when compared to the control group. In addition, there was a significant reduction of body weight $(p<0.001)$ in the SMBG group [50]. Key studies of SMBG in types 1 and 2 DM are summarized in Table 2.

SMBG is essential in the management of type $1 \mathrm{DM}$ patients and those patients with type $2 \mathrm{DM}$ who are on insulin. Also, there is emerging evidence to support the use of SMBG in type 2 patients on non-insulin therapy.

\section{Emerging technologies}

Although, currently, SMBG is the simplest and the most practical method of blood glucose monitoring, it is also important to consider the emerging technologies. Goals for future techniques include noninvasive monitoring and more comprehensive blood glucose data collection.
The newer technologies include real-time CGM, flash glucose monitoring, Bluetooth-enabled meter, diabetes apps, glucose-sensing contact lens and Ambulatory Glucose Profile (AGP) by Free style Libre [91, 92]. Detailed discussion of these technologies at this point of time does not appear relevant to the consensus process and therefore is not included in this document. 


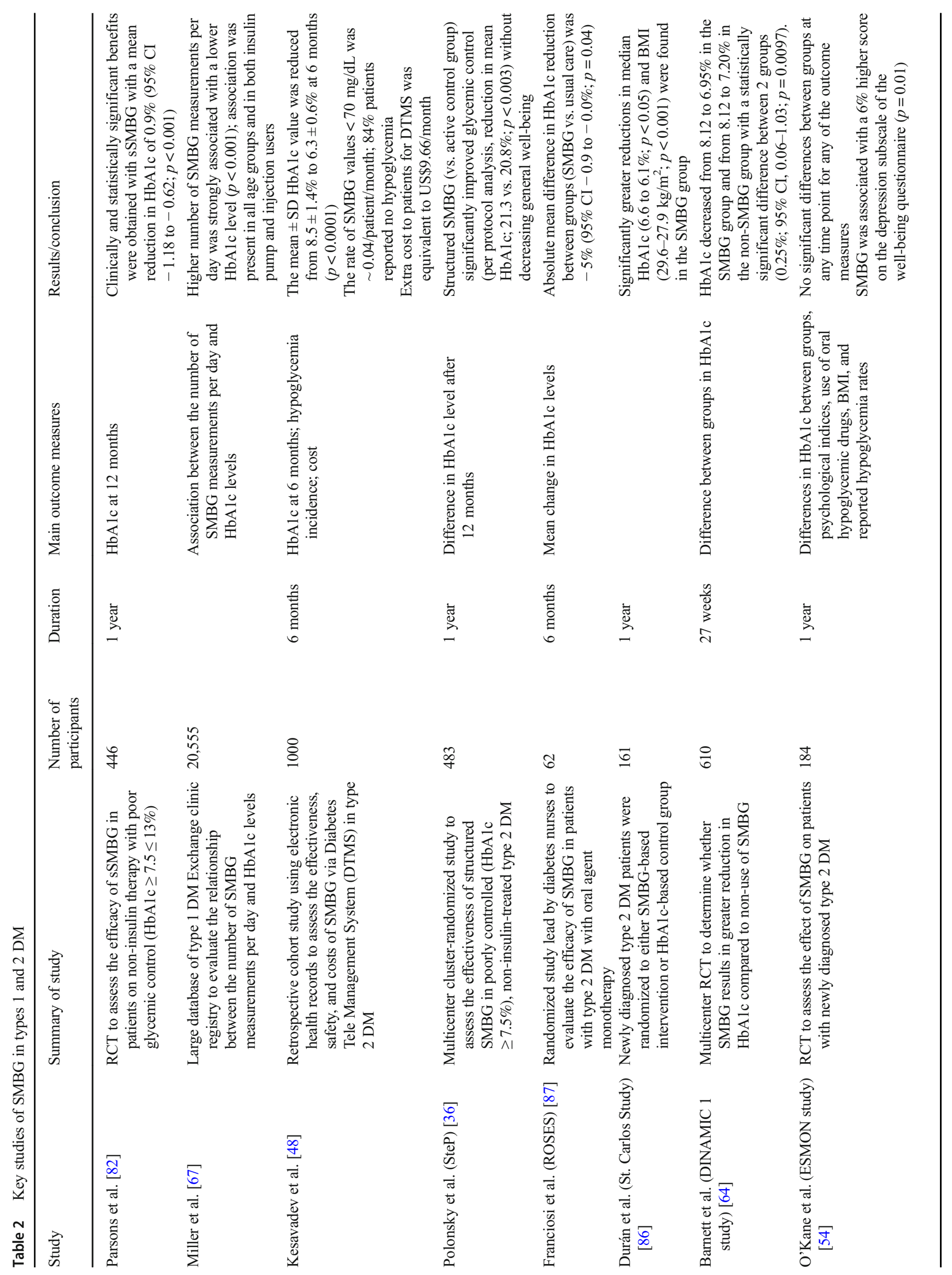




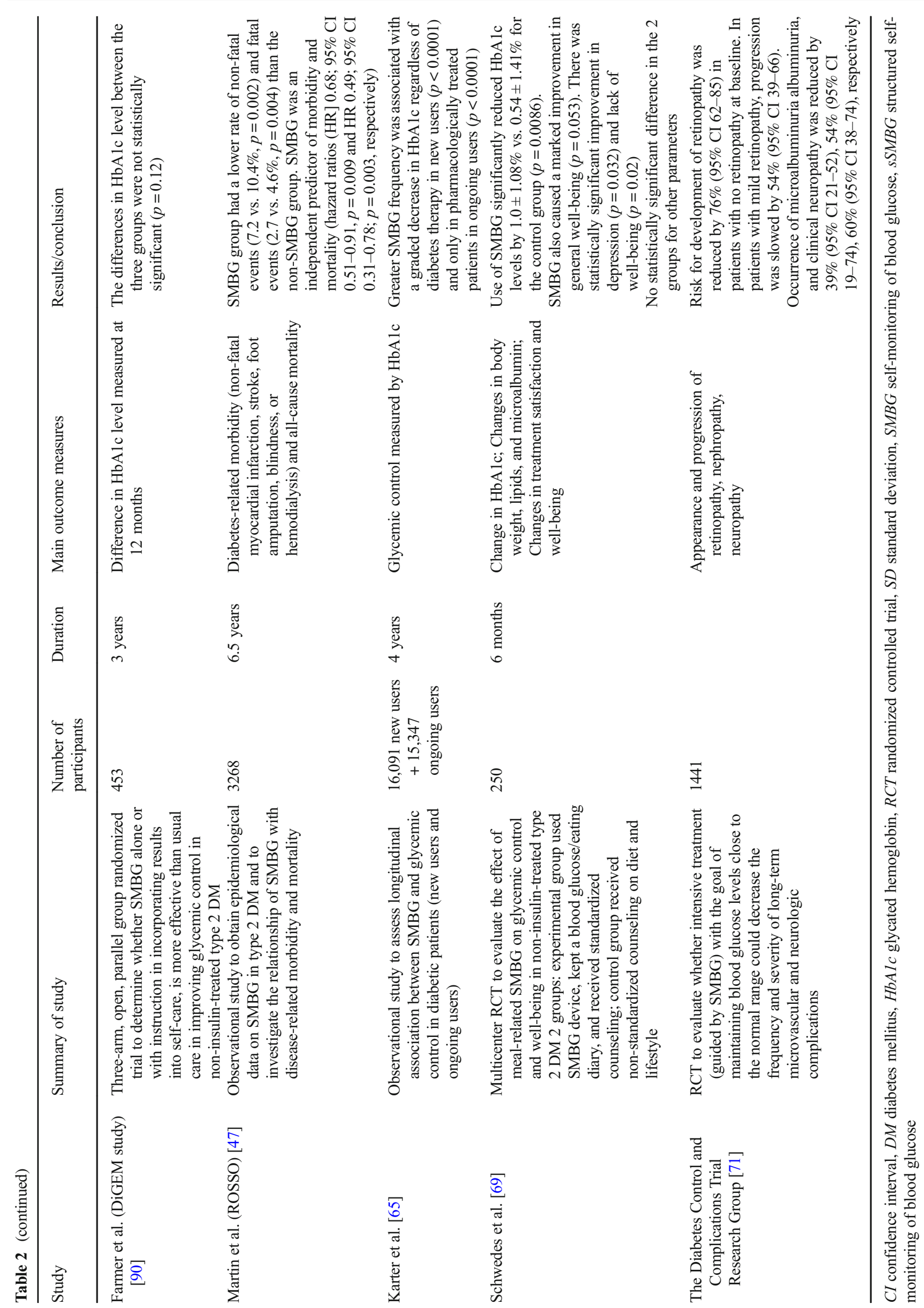




\section{What do the RSSDI recommendations on SMBG say?}

Research Society for the Study of Diabetes in India (RSSDI) recently (2017) published the clinical practice recommendations for the management of type $2 \mathrm{DM}$. These guidelines also include a section on SMBG. RSSDI provides two levels of recommendations: "Recommended care" and "Limited care." As per the RSSDI, recommended care [93]:

- SMBG is useful to people with diabetes who have the required knowledge, skills, and willingness to use the information obtained through testing to actively adjust treatment with the help of the treating physician and to enhance understanding of diabetes and assess the effectiveness of the management plan on glycemic control.

- The purpose of performing SMBG and using SMBG data should be agreed between the person with diabetes and the healthcare provider.

- SMBG should be available on an ongoing basis to those using insulin.

- SMBG protocols (intensity and frequency) should be individualized to address each individual's specific educational/ behavioral/clinical requirements, specific needs, and goals (to identify/prevent/manage acute hyper- and hypoglycemia) and provider requirements for data on glycemic patterns and to monitor impact of therapeutic decision-making.

- Intensive/regular SMBG may be recommended in patients on multiple daily insulin injections, in case of pre-gestational/gestational diabetes on insulin, history of hypoglycemia unawareness, brittle diabetes, or with poor metabolic control on multiple oral antidiabetic agents (OADs) and/or basal insulin.

- SMBG should be performed at least as often as insulin is administered. Patients on intensive insulin regimens who are on multiple doses of insulin or on insulin pumps should be tested three or more times daily (all pre-meals, post-meals, bedtime, prior to exercise).

- SMBG plays an important role when low blood glucose is suspected or after treating low blood glucose until normoglycemia is achieved and prior to critical tasks such as driving. For many patients, this will require testing 6-10 (or more) times daily, although individual needs may vary.

- Pregnant women with insulin-treated diabetes should be advised to perform SMBG on a daily basis, failing which, at least weekly monitoring should be encouraged.

- Ideal SMBG is seven tests/day, i.e., three before and three after each meal and one test at 3 a.m. If this is not feasible, one fasting test and three tests each after breakfast, lunch, and dinner daily may be done, which can further be individualized to twice or thrice a week as the pregnancy advances.
- More frequent monitoring should be done in special situations like fever, vomiting, and persistent polyuria with uncontrolled blood glucose, especially if abdominal pain or rapid breathing is present. Ketone test should be performed as and when needed.

- SMBG accuracy is instrument and user-dependent, so it is important to evaluate each patient's monitoring technique, both initially and at regular intervals thereafter. The ongoing need for and frequency of SMBG should be reevaluated at each routine visit.

- SMBG should be considered for people using oral glucoselowering medications as an optional component of selfmanagement and in association with $\mathrm{HbAlc}$ testing:

- To provide information on, and help avoid, hypoglycemia

- To assess changes in blood glucose control due to medications and lifestyle changes

- To monitor the effects of foods on post-prandial glycemia

- To monitor changes in blood glucose levels during intercurrent illness

- SMBG may be useful in type $2 \mathrm{DM}$ during periods of acute illness; in patients using sulfonylureas or glinides as combination or monotherapy; to identify hypoglycemia especially in the first 3 months of starting sulfonylurea; in patients who experience episodes of hypoglycemia and who have reduced awareness of hypoglycemia; in drivers and those who fast; and in women under preconception care.

- Regular use of SMBG should not be considered part of routine care where diabetes is well-controlled by nutrition therapy or oral medications alone.

- Structured assessment of self-monitoring skills, the quality and use made of the results obtained, and of the equipment used should be made annually.

RSSDI recommendations for limited care state "SMBG using meters with strips should be considered for people with diabetes using insulin or drugs like sulfonylurea and glinides." Table 3 shows RSSDI recommendations for target blood glucose levels in patients with DM.

Table 3 Target blood glucose levels in patients with DM as per RSSDI recommendations [93]

\begin{tabular}{ll}
\hline & HbAlc \\
\hline Target & $<7.0 \%$ \\
Fasting plasma glucose $(\mathrm{mg} / \mathrm{dL})$ & $\leq 115$ \\
Post-prandial glucose $(\mathrm{mg} / \mathrm{dL})$ & $\leq 160$ \\
\hline
\end{tabular}




\section{Diabetes in pregnancy}

Gestational DM (GDM) and pre-existing DM in pregnant women are associated with increased risk of perinatal morbidity and mortality. A common complication is macrosomia or large-for-gestational-age babies. The hyperglycemia and adverse pregnancy outcomes (HAPO) study found that there is a strong association of maternal hyperglycemia (of a level lesser than that diagnostic of diabetes) with increased birth weight and increased cord-blood serum C-peptide levels [94]. Proper management can reduce the risk of maternal and neonatal complications and improve outcomes [95].

All women with pre-existing DM should receive prepregnancy counseling, which should include explaining the risks and common complications and strategies to minimize them [96]. As per the IDF, women who are on insulin should be advised on maintaining $\mathrm{HbA} 1 \mathrm{c}$ level below 6.5 or $7.0 \%$. If HbA1c is above $8.0 \%$, women should be discouraged from becoming pregnant until the glycemic control can be improved [96]. A meta-analysis showed that pre-pregnancy care for women with pre-gestational type 1 or $2 \mathrm{DM}$ improves rates of congenital malformations, perinatal mortality, and reduces maternal HbAlc in the first trimester of pregnancy [97].

Maintaining a tight blood glucose control is essential in pregnancy and, therefore, SMBG plays an important role [98]. Government of India, in the recently published revised guidelines on diagnosis and management of GDM, has recommended target fasting blood glucose as less than $95 \mathrm{mg} / \mathrm{dL}$ and all 2-h post-prandial glucose levels as less than $120 \mathrm{mg} / \mathrm{dL}$ [99]. Women with pre-existing type $2 \mathrm{DM}$ from central India have shown to have significantly higher post dinner blood glucose than post breakfast [100]. Thus, women on insulin therapy should do frequent testing including fasting, 2-h post breakfast, 2-h post lunch, and 2-h post dinner for insulin dose adjustment. The IDF guidelines also advise women with GDM to perform SMBG four times daily (fasting and $1 \mathrm{~h}$ after each meal) [96].

\section{Frequency and timing of SMBG}

A consensus on the frequency and timing of SMBG has not yet been established. Different SMBG regimens should be followed based on factors such as diabetes type, treatment approach (diet, oral antidiabetic medication, or insulin), glycemic control, available resources, and patient's level of education. While patients on intensive insulin regimens may require up to 10 tests daily, patients on diet and oral medication may only need 6 to 8 tests per week $[73,93,101]$.

The IDF guideline for non-insulin-treated type 2 DM describes focused and low-intensity SMBG regimens. Focused regimens include the 5- and 7-point profiles in which blood glucose is measured 5 or 7 times a day, respectively, for 3 consecutive days [102]. Another focused regimen is the staggered regimen in which blood glucose levels are measured pre- and post-meal (two tests per day) for alternating meal over a period of 1 week. Low-intensity SMBG regimens include meal-based testing (before and after selected meals), detection/assessment fasting hyperglycemia (bedtime and morning fasting SMBG), and detection of asymptomatic hypoglycemia (pre-lunch and pre-supper SMBG) [102].

In 2011, a group of experts in diabetology and endocrinology recommended two schemes for SMBG in type $2 \mathrm{DM}$, one for less intensive testing and the other for intensive testing. The less intensive testing focusses on paired testing (pre- and post-prandial) once per day. The duration of the paired testing could be 1/month, 1 week/month, 3-7 days/week, or continuous daily testing depending on individual requirement. Intensive testing involves seven tests per day over a period of 3 to 7 days. The duration could be 3 days/week to continuous daily monitoring [101].

In an Indian publication, the authors recommend blood glucose checks at least three times daily in patients with type 1 DM. They recommend a check of pre-meal blood glucose initially until the target pre-prandial levels are reached, after which post-meal levels can be checked. Thus, they divide the SMBG regimen for type 1 DM in 2 phases, "Initial phase" and "Optimization phase." For type 2 DM, they recommend different regimens; for example, multiple tests per day regimen, and staggered regimen. For type 2 patients on intensive insulin regimens, they advise monitoring similar to patients with type $1 \mathrm{DM}$, and less intensive monitoring for other patients. For those with $\mathrm{HbAlc}$ above target, they advise testing at least twice daily, and for those with $\mathrm{HbA1c}$ on target at least 4 times per week (at different times each day) [103].

SMBG frequency and timing vary depending on the diabetes type, treatment approach, glycemic control, available resources, and patient's level of education.

\section{SMBG practice in India and unmet need for country-specific guidelines and tool}

Burden of DM in India is very high and it is projected to get worse in the coming years. SMBG, with its potential to help in achieving good glycemic control and reducing the risk of both short-and long-term complications, can serve as an apt measure to deal with DM. While SMBG is widely used in other parts of the world, it is less commonly practiced in India. The SMBG International Working Group, in 2008, conducted a 
Table 4 Estimated SMBG use in different countries [62, 65, 105-108]

\begin{tabular}{ll}
\hline Country (study year) & SMBG use (\%) \\
\hline Canada (2013) & 87.8 \\
Australia (2006) & 70 \\
USA (2006) & 62.2 \\
India (middle-/high-income & 28.4 \\
population) (2006) & 15.3 \\
Malaysia (2007) & 11 \\
India (2004) & \\
\hline
\end{tabular}

survey to study the use of SMBG in 13 countries including India. The lowest use of SMBG was found in India $(0.2 \%)$ [104]. A study conducted in Delhi to evaluate the quality of care in patients from the middle- and high-income group found that $28.4 \%$ of the patients had a home blood glucose monitoring device, and $77.4 \%$ of the patients were following the advice on SMBG [105]. Table 4 shows the estimated SMBG use in different countries.

Even when used, the process of SMBG seems to be far from ideal in India. Recently, a survey was conducted in Chennai to understand the knowledge and practice of SMBG in patients with type 2 DM performing SMBG at home. Sadly, only a quarter of the survey participants had adequate knowledge of the process of SMBG and were following the procedure appropriately [109]. This could be due to lack of education about the purpose of SMBG and the correct process to be followed. The current use of SMBG in India appears to be mostly "random," without a structured process. The importance of education and practice of "structured" SMBG cannot be overemphasized, especially in the Indian setting.

In spite of being aware of the importance of SMBG for glycemic control in patients with diabetes, primary care physicians may not have the expertise to develop an appropriate plan for their patients. Availability of an easy tool that can be applied for different clinical scenarios will be very useful in such a setting. It is, needless to say, that India has several factors such as availability of healthcare resources, spending capacity of patients, education level of patients (to understand the intricacies of SMBG), and patient beliefs, that are different from those in other parts of the world. Therefore, it is imperative to develop a tool that is easy to understand and can be implemented with ease in the Indian context.

\section{Consensus methodology}

In order to fulfill this unmet need, a panel of expert endocrinologists/diabetologists came together under the aegis of RSSDI, reviewed the current literature, combined the evidences with their clinical knowledge and expertise, and developed the first draft for the consensus recommendations/guidelines/tools to be followed for SMBG in India. The expert panel included members of executive committee of RSSDI and invited key opinion leaders (KOLs) from across the country representing government as well as private institutions.

The first draft was circulated among the expert panel members for their critical comments and suggestions for amendments. All the relevant feedback and suggestions were included in the revised draft and it was circulated to the panel for second review and feedback was also sought for different SMBG tools which were circulated in the form of questionnaires. This was followed by the expert committee meeting held on 4 March 2018 in Mumbai where the revised consensus draft was discussed page by page and lot of important suggestions came in for the improvement of the consensus recommendations. The revised draft for SMBG consensus recommendations was circulated again to the expert panel for review and suggestions and was further circulated to extended group for critical feedback and suggestions. The final document after revision was presented at the RSSDI executive committee meeting on 7 April at Jaipur and was formally adopted by RSSDI and sent for publication to the International Journal of Diabetes in Developing Countries (IJDDC).

Current SMBG practices is India are not ideal. Proper education and a simple tool, which will be easy to be followed and implemented is necessary. A panel of experts was convened to fulfill this unmet need.

\section{Recommendations by the expert panel}

The expert panel has set the following basic definitions:

Well-controlled diabetes
Patients who are within RSSDI recommended target range of blood glucose levels and $\mathrm{HbA1c}$.
Uncontrolled diabetes/ Patients who are outside the RSSDI poorly controlled diabetes Brittle diabetes recommended target range of blood glucose levels and $\mathrm{HbAlc}$. Diabetes that is difficult to control, with severe instability of blood glucose levels and with frequent and unpredictable episodes of 


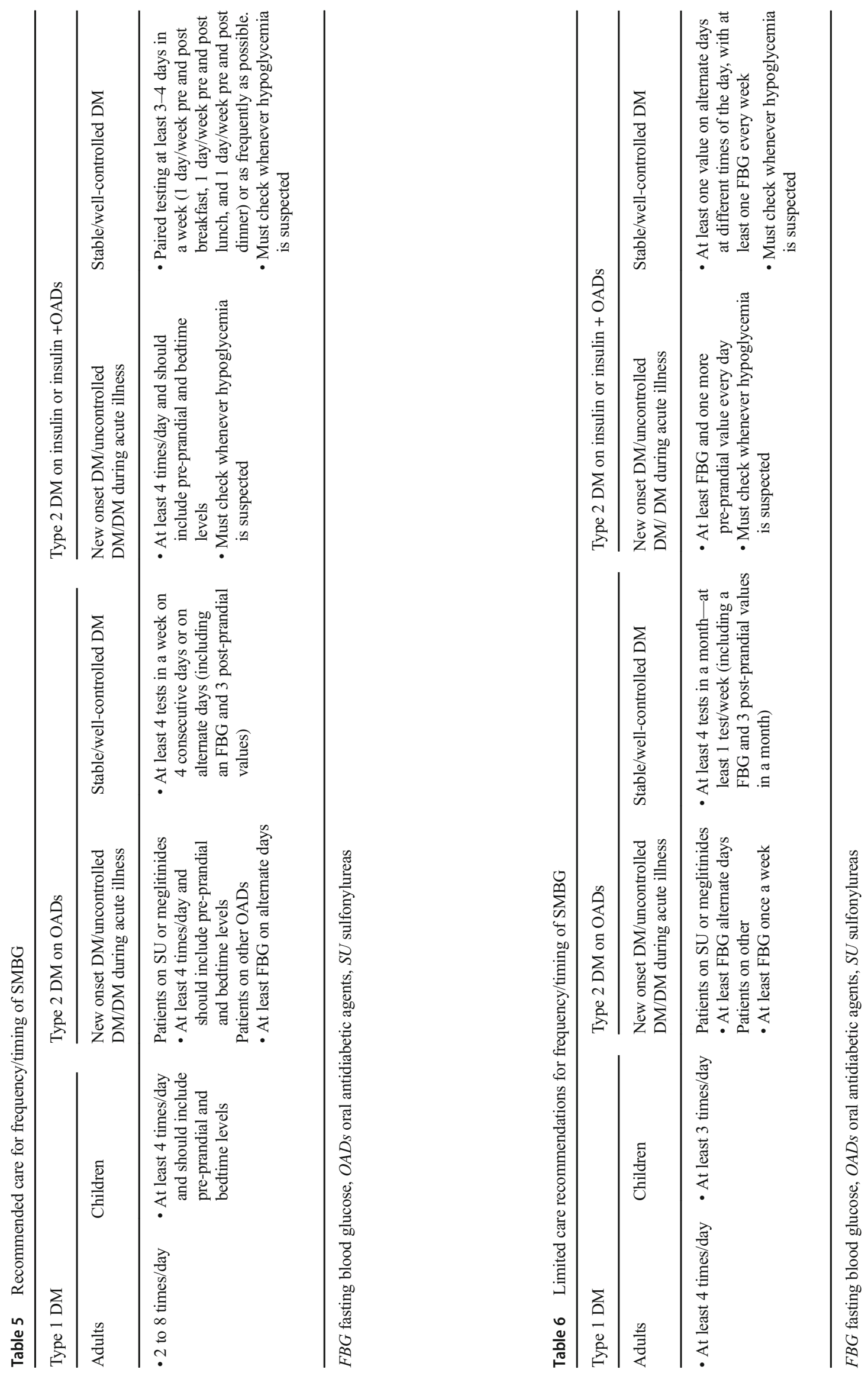


Table 7 Recommended care and limited care for frequency/timing of SMBG for diabetes in pregnancy

\begin{tabular}{|c|c|c|c|}
\hline \multicolumn{2}{|l|}{ Patients on lifestyle modifications } & \multicolumn{2}{|l|}{ Patients on OADs or insulin } \\
\hline Recommended care & Limited care & Recommended care & Limited care \\
\hline $\begin{array}{l}\text { - A day profile once a week-FBG } \\
\text { and } 3 \text { post-prandial values at least } \\
\text { once a week or staggered over the } \\
\text { week }\end{array}$ & $\begin{array}{l}\text { - } 1 \text { FBG and one post-prandial value } \\
\text { every week (any meal, preferably } \\
\text { largest meal of the day) }\end{array}$ & $\begin{array}{l}\text { - At least } 4 \text { times/day (FBG } \\
\text { and } 3 \text { post-prandial values) }\end{array}$ & $\begin{array}{l}\text { - Paired testing every day (pre- and } \\
\text { post-breakfast on 1st day, pre- and } \\
\text { post-lunch on 2nd day, pre- and } \\
\text { post-dinner on 3rd day, and then } \\
\text { keep repeating the cycle) }\end{array}$ \\
\hline
\end{tabular}

$F B G$ fasting blood glucose

hypoglycemia and/or ketoacidosis, which lead to disruption of quality of life.

New onset diabetes

Newly diagnosed diabetes

Recommended care

Recommended care constitutes evidence-based care which is costeffective.

Limited Care Limited care is the lowest level of care that seeks to achieve the major objectives of diabetes management provided in healthcare settings with very limited resources such as drugs, personnel, technologies, and procedures.

These recommendations by the expert panel include details of the SMBG regimens for different clinical scenarios. These recommendations conform with and can be considered as an extension of the recently published RSSDI recommendations, in which SMBG regimens were not discussed in detail [93].

\section{General recommendations}

- $\quad$ RSSDI-recommended target levels should be adequately explained to the patient/provider and mutually agreed between the patient/provider and the clinician.

- SMBG technique should be properly explained to the patient.

Table 8 Recommended schedule for patients on basal insulin
- SMBG technique of the patients should be evaluated regularly and appropriate feedback given.

- $\quad$ SMBG device should comply with the ISO 15197:2013 requirements.

- The recommended target levels that should be followed for most diabetes patients for fasting blood glucose, postprandial blood glucose, and HbA1c are $\leq 115 \mathrm{mg} / \mathrm{dL}, \leq$ $160 \mathrm{mg} / \mathrm{dL}$, and $<7.0 \%$, respectively [93].

- Patients should be educated that the post-prandial blood glucose levels should be checked after $1 / 2 \mathrm{~h}$ from the start of the meal and not the end of the meal.

- Patients may be allowed to make minor adjustments to insulin dosage and changes in diet and exercise based on the SMBG readings.

- Annual structured assessment should be carried out to evaluate patient's self-monitoring skills including monitoring technique, interpretation of blood glucose results, impact on patient's quality of life, and continued benefit to the patient (a questionnaire will be developed for annual evaluation of the patients).

\section{Recommendations for use of lancets/pricking devices}

Recommended care Single use of lancet/pricking needles (disposable injection needles are commonly used in India in place of lancets) is recommended.

\begin{tabular}{|c|c|c|c|c|c|c|c|c|}
\hline & $\begin{array}{l}\text { Fasting/pre- } \\
\text { breakfast }\end{array}$ & $\begin{array}{l}\text { Post- } \\
\text { breakfast }\end{array}$ & $\begin{array}{l}\text { Pre- } \\
\text { lunch }\end{array}$ & $\begin{array}{l}\text { Post- } \\
\text { lunch }\end{array}$ & $\begin{array}{l}\text { Pre- } \\
\text { dinner }\end{array}$ & $\begin{array}{l}\text { Post- } \\
\text { dinner }\end{array}$ & 3 a.m. & $\mathrm{SOS}^{\dagger}$ \\
\hline Monday & $\checkmark$ & & & & & & & \\
\hline Tuesday & $\checkmark$ & & & & & & & \\
\hline Thursday & $\checkmark$ & & & & & & & \\
\hline Friday & $\checkmark$ & & & & & & & \\
\hline Saturday & $\checkmark$ & & & & & & & \\
\hline Sunday & $\checkmark$ & & & & & & & \\
\hline
\end{tabular}

Limited care: fasting levels twice a week or once in 3 days

After achievement of fasting target, post-prandial correction should be done

${ }^{\dagger}$ SOS whenever hypoglycemia is suspected and during intercurrent acute illness 
Table 9 Recommended schedule for patients on premix insulin, basal bolus therapy

\begin{tabular}{llllllll}
\hline & $\begin{array}{l}\text { Fasting/pre- } \\
\text { breakfast }\end{array}$ & $\begin{array}{l}\text { Post- } \\
\text { breakfast }\end{array}$ & $\begin{array}{l}\text { Pre- } \\
\text { lunch }\end{array}$ & $\begin{array}{l}\text { Post- } \\
\text { lunch }\end{array}$ & $\begin{array}{l}\text { Pre- } \\
\text { dinner }\end{array}$ & $\begin{array}{l}\text { Post- } \\
\text { dinner }\end{array}$ & 3 a.m. \\
\hline $\begin{array}{l}\text { Monday } \\
\text { Tuesday } \\
\text { Wednesday }\end{array}$ & $\checkmark$ & $\checkmark$ & $\checkmark$ & $\checkmark$ & $\checkmark$ & $\checkmark$ \\
$\begin{array}{l}\text { Thursday } \\
\text { Friday } \\
\text { Saturday }\end{array}$ & $\checkmark$ & $\checkmark$ & $\checkmark$ & $\checkmark$ & $\checkmark$ & $\checkmark$ \\
Sunday & $\checkmark$ & $\checkmark$ & $\checkmark$ & $\checkmark$ & $\checkmark$ & $\checkmark$ \\
\hline
\end{tabular}

Once target is achieved, less frequent testing can be done; fasting and one meal-related testing can be done, can be staggered (changing every 2 days)

${ }^{\dagger}$ SOS whenever hypoglycemia is suspected and during intercurrent acute illness
Limited care Although single use is recommended, it is important to also consider the cost especially in limited resource settings. It is recommended that if a patient chooses to reuse the lancet or pricking device, proper antiseptic precautions should be taken. The lancet/pricking device should be discarded when the tip goes blunt or the prick becomes painful. Also, the lancet/pricking device should be immediately discarded if it comes in contact with another individual's blood. If a patient decides to reuse pricking needle, proper care must be taken as mentioned below:

- Cover should be placed back on the needle immediately.

- Needle should not touch any surface apart from the inside of the needle cover.

- Cleaning the needle with alcohol should be avoided as it can make the point blunt.

\section{Recommendations based on DM type, treatment approach, and glycemic control}

The expert panel recommends customizing the frequency and timing of SMBG depending on whether it is type 1 or $2 \mathrm{DM}$. In patients with type $2 \mathrm{DM}$, monitoring will further vary depending on whether the patient is on OADs or insulin and whether it is new onset DM/uncontrolled DM or well-controlled DM. The panel provides recommendations for two levels of care: recommended care and limited care (Tables 5 and 6 ).

All patients on multiple-dose insulin therapy should perform SMBG at least two times/day (ideally before any insulin injection). More frequent testing may be required in:

- Patients with frequent hypoglycemia or hypoglycemic symptoms

- Patients not at HbAlc target levels

In patients on intensive insulin therapy, blood glucose levels should be checked at fasting, pre-meal, at bedtime, and periodically at $3 \mathrm{am}$.

\section{Recommendations for diabetes in pregnancy}

In patients with pre-existing diabetes or GDM, target blood glucose levels should be 70 to $90 \mathrm{mg} / \mathrm{dL}$ fasting, $<140 \mathrm{mg} / \mathrm{dL} \mathrm{1-h}$ post-prandial, and $<120 \mathrm{mg} / \mathrm{dL}$ 2-h post-prandial. Patients on lifestyle modifications should have a day profile once a week.
Table 10 Recommended Schedule for patients with brittle diabetes and hypoglycemia unawareness

\begin{tabular}{llllllll}
\hline & $\begin{array}{l}\text { Fasting/ } \\
\text { prebreakfast }\end{array}$ & $\begin{array}{l}\text { Post- } \\
\text { breakfast }\end{array}$ & $\begin{array}{l}\text { Pre- } \\
\text { lunch }\end{array}$ & $\begin{array}{l}\text { Post- } \\
\text { lunch }\end{array}$ & $\begin{array}{l}\text { Pre- } \\
\text { dinner }\end{array}$ & $\begin{array}{l}\text { Post- } \\
\text { dinner }\end{array}$ & 3 \\
\hline a.m.* & SOS $^{\dagger}$ \\
Tuesday & $\checkmark$ & $\checkmark$ & $\checkmark$ & $\checkmark$ & $\checkmark$ & $\checkmark$ & \\
Wednesday & $\checkmark$ & $\checkmark$ & $\checkmark$ & $\checkmark$ & $\checkmark$ & $\checkmark$ & \\
Thursday & $\checkmark$ & $\checkmark$ & $\checkmark$ & $\checkmark$ & $\checkmark$ & $\checkmark$ & $\checkmark$ \\
Friday & $\checkmark$ & $\checkmark$ & $\checkmark$ & $\checkmark$ & $\checkmark$ & $\checkmark$ & \\
Saturday & $\checkmark$ & $\checkmark$ & $\checkmark$ & $\checkmark$ & $\checkmark$ & $\checkmark$ & \\
Sunday & $\checkmark$ & $\checkmark$ & $\checkmark$ & $\checkmark$ & $\checkmark$ & $\checkmark$ & \\
\hline
\end{tabular}

*3 a.m. testing should be done at least once a week

${ }^{\dagger}$ SOS whenever hypoglycemia is suspected or during intercurrent acute illness. SMBG may not be ideal in this case and CGM may be required 
Table 11 Glycemic targets in elderly patients as per ADA and AGS consensus [110]

\begin{tabular}{llll}
\hline Target glycemic levels & Healthy elderly & $\begin{array}{l}\text { Elderly with intermediate } \\
\text { health status }\end{array}$ & $\begin{array}{l}\text { Elderly with poor } \\
\text { health status }\end{array}$ \\
\hline HbAlc & $<7.5 \%$ & $<8.0 \%$ & $<8.5 \%$ \\
Fasting or pre-prandial glucose (mg/dL) & $90-130$ & $90-150$ & $100-180$ \\
Bedtime glucose (mg/dL) & $90-150$ & $100-180$ & $110-200$ \\
\hline
\end{tabular}

This should include one fasting and three post-prandial values at least once a week or staggered over a week (this is consensus opinion, not based on published evidence) (Table 7). Patients on OADs or insulin should perform intensive monitoring.

\section{Recommendations by the expert panel for patients on basal insulin}

In patients on basal insulin, daily fasting levels are recommended (recommended care) (Table 8). In resource-limited settings, fasting levels can be performed twice a week or once in 3 days (limited care). Post-prandial correction should be done after correcting fasting blood glucose.

\section{Recommendations by the expert panel for premix insulin or basal bolus}

Patients on premix insulin or basal bolus therapy should be advised to perform three pre-prandial (including fasting) and three post-prandial tests on alternate days till target $\mathrm{HbAlc}$ and blood glucose levels are reached. After achievement of the target, less frequent testing can be done (Table 9).

\section{Recommendations by the expert panel for patients with brittle diabetes and hypoglycemia unawareness}

In patients with brittle diabetes or hypoglycemia unawareness, 7-point testing is recommended with a 3 a.m. testing at least once a week (Table 10).

\section{Special situations/hemodynamically unstable conditions/end stage organ disease}

These patients are usually on multiple doses of insulin per day or on insulin infusions. In these patients, the frequency or timing of SMBG should be customized based on the individual case. More frequent monitoring may be required based on the clinical situation.

\section{Recommendations for elderly patients}

In elderly patients, monitoring should be less frequent, and the target should be relaxed to avoid hypoglycemia. A consensus by the American Diabetic Association and the American Geriatrics Society recommends dividing the patients into three categories based on their health status to enable customizing the glycemic targets. Their recommendations are listed in Table 11 [110]. The expert panel endorses these recommendations for glycemic targets in the elderly.

The expert panel recommends that, in the initiation phase, the frequency of SMBG should be once daily (different time each day) and later it should be reduced further to two to three times per week (Table 12). Hypoglycemia is a special concern in the elderly and pre-prandial values are important. The family should also be educated and trained on SMBG.

The expert panel hopes that these consensus recommendations will serve as a valuable tool for the practice of SMBG in India.
Table 12 Recommended schedule for elderly patients

\begin{tabular}{|c|c|c|c|c|c|c|c|c|}
\hline & $\begin{array}{l}\text { Fasting/pre- } \\
\text { breakfast }\end{array}$ & $\begin{array}{l}\text { Post- } \\
\text { breakfast }\end{array}$ & $\begin{array}{l}\text { Pre- } \\
\text { lunch }\end{array}$ & $\begin{array}{l}\text { Post- } \\
\text { lunch }\end{array}$ & $\begin{array}{l}\text { Pre- } \\
\text { dinner }\end{array}$ & $\begin{array}{l}\text { Post- } \\
\text { dinner }\end{array}$ & 3 a.m. & $\mathrm{SOS}^{\dagger}$ \\
\hline Monday & $\checkmark$ & & & & & & & \\
\hline Tuesday & & $\checkmark$ & & & & & & \\
\hline Wednesday & & & $\checkmark$ & & & & & \\
\hline Thursday & & & & $\checkmark$ & & & & \\
\hline Friday & & & & & $\checkmark$ & & & \\
\hline Saturday & & & & & & $\checkmark$ & & \\
\hline Sunday & $\checkmark$ & & & & & & & \\
\hline
\end{tabular}

The above regimen is for initiation phase. Once target is achieved, frequency should be reduced to 2 to 3 tests/ week

${ }^{\dagger}$ SOS whenever hypoglycemia is suspected or during intercurrent acute illness 
Acknowledgements The authors thank Turacoz Healthcare Solutions (www.Turacoz.com), Gurugram, India, for writing support.

\section{Compliance with ethical standards}

Conflict of interest This consensus was supported by restricted academic grant from Roche. However, there was no interference/influence from Roche in the consensus development. None of the authors have declared any conflict of interest.

Open Access This article is distributed under the terms of the Creative Commons Attribution 4.0 International License (http:// creativecommons.org/licenses/by/4.0/), which permits unrestricted use, distribution, and reproduction in any medium, provided you give appropriate credit to the original author(s) and the source, provide a link to the Creative Commons license, and indicate if changes were made.

\section{References}

1. Narayan KM, Gregg EW, Fagot-Campagna A, Engelgau MM, Vinicor F. Diabetes - a common, growing, serious, costly, and potentially preventable public health problem. Diabetes Res Clin Pract. 2000;50(Suppl 2):S77-84.

2. Yesudian CA, Grepstad M, Visintin E, Ferrario A. The economic burden of diabetes in India: a review of the literature. Glob Health. 2014;10:80

3. Marín-Peñalver JJ, Martín-Timón I, Sevillano-Collantes C, Del Cañizo-Gómez FJ. Update on the treatment of type 2 diabetes mellitus. World J Diabetes. 2016;7(17):354-95.

4. Scambler S, Newton P, Asimakopoulou K. The context of empowerment and self-care within the field of diabetes. Health (London). 2014;18(6):545-60.

5. International Diabetes Federation. IDF Diabetes Atlas $8^{\text {th }}$ Edition. Available at: http://www.diabetesatlas.org/. Accessed on $24^{\text {th }}$ November 2017

6. Nakagami T, Qiao Q, Carstensen B, Nhr-Hansen C, Hu G, Tuomilehto J, et al. Age, body mass index and type 2 diabetesassociations modified by ethnicity. Diabetologia. 2003;46(8): 1063-70.

7. Mohan V. Why are Indians more prone to diabetes? J Assoc Physicians India. 2004;52:468-74.

8. Joshi SR. Diabetes care in India. Ann Glob Health. 2015;81(6): 830-8.

9. Rahman S, Rahman T, Ismail AA, Rashid AR. Diabetesassociated macrovasculopathy: pathophysiology and pathogenesis. Diabetes Obes Metab. 2007;9(6):767-80.

10. Acharya LD, Rau NR, Udupa N, Rajan MS, Vijayanarayana K. Assessment of cost of illness for diabetic patients in south Indian tertiary care hospital. J Pharm Bioallied Sci. 2016;8(4):314-20.

11. Bhansali A. Cost of diabetes care: prevent diabetes or face catastrophe. J Assoc Physicians India. 2013;61(2):95.

12. Kumpatla S, Kothandan H, Tharkar S, Viswanathan V. The costs of treating long-term diabetic complications in a developing country: a study from India. J Assoc Physicians India. 2013;61(2):102-9.

13. Stratton IM, Adler AI, Neil HA, Matthews DR, Manley SE, Cull $\mathrm{CA}$, et al. Association of glycaemia with macrovascular and microvascular complications of type 2 diabetes (UKPDS 35): prospective observational study. BMJ. 2000;321(7258):405-12.

14. Vasudevan AR, Burns A, Fonseca VA. The effectiveness of intensive glycemic control for the prevention of vascular complications in diabetes mellitus. Treat Endocrinol. 2006;5(5):273-86.
15. Cavalot F. Do data in the literature indicate that glycaemic variability is a clinical problem? Glycaemic variability and vascular complications of diabetes. Diabetes Obes Metab. 2013;15(Suppl 2):3-8.

16. Ohkubo $\mathrm{Y}$, Kishikawa H, Araki E, Miyata T, Isami S, Motoyoshi $\mathrm{S}$, et al. Intensive insulin therapy prevents the progression of diabetic microvascular complications in Japanese patients with noninsulin-dependent diabetes mellitus: a randomized prospective 6year study. Diabetes Res Clin Pract. 1995;28(2):103-17.

17. Leal J, Hayes AJ, Gray AM, Holman RR, Clarke PM. Temporal validation of the UKPDS outcomes model using 10-year posttrial monitoring data. Diabetes Care. 2013;36(6):1541-6.

18. UK Prospective Diabetes Study (UKPDS) Group. Effect of intensive blood-glucose control with metformin on complications in overweight patients with type 2 diabetes (UKPDS 34). Lancet. 1998;352(9131):854-65.

19. UK Prospective Diabetes Study (UKPDS) Group. Intensive blood-glucose control with sulphonylureas or insulin compared with conventional treatment and risk of complications in patients with type 2 diabetes (UKPDS 33). Lancet. 1998;352(9131):837-53.

20. Diabetes Control and Complications Trial (DCCT)/Epidemiology of Diabetes Interventions and Complications (EDIC) Study Research Group. Intensive diabetes treatment and cardiovascular outcomes in type 1 diabetes: the DCCT/EDIC study 30-year follow-up. Diabetes Care. 2016;39(5):686-93.

21. Kirk JK, Stegner J. Self-monitoring of blood glucose: practical aspects. J Diabetes Sci Technol. 2010;4(2):435-9.

22. Vashist SK. Continuous glucose monitoring systems: a review. Diagnostics (Basel). 2013;3(4):385-412.

23. Little RR, Rohlfing CL. The long and winding road to optimal HbA1c measurement. Clin Chim Acta. 2013;418:63-71.

24. Kesavadev J, Sadikot S, Wangnoo S, Kannampilly J, Saboo B, Aravind SR, et al. Consensus guidelines for glycemic monitoring in type 1/type $2 \&$ GDM. Diabetes Metab Syndr. 2014;8(3):187-95.

25. Nalysnyk L, Hernandez-Medina M, Krishnarajah G. Glycaemic variability and complications in patients with diabetes mellitus: evidence from a systematic review of the literature. Diabetes Obes Metab. 2010;12(4):288-98.

26. Zinman B, Marso SP, Poulter NR, Emerson SS, Pieber TR, Pratley $\mathrm{RE}$, et al. Day-to-day fasting glycaemic variability in DEVOTE: associations with severe hypoglycaemia and cardiovascular outcomes (DEVOTE 2). Diabetologia. 2018;61(1):48-57.

27. Liang S, Yin H, Wei C, Xie L, He H, Liu X. Glucose variability for cardiovascular risk factors in type 2 diabetes: a meta-analysis. $\mathrm{J}$ Diabetes Metab Disord. 2017;16:45.

28. Lin CC, Yang CP, Li CI, Liu CS, Chen CC, Lin WY, et al. Visit-tovisit variability of fasting plasma glucose as predictor of ischemic stroke: competing risk analysis in a national cohort of Taiwan diabetes study. BMC Med. 2014;12:165.

29. Rizzo MR, Marfella R, Barbieri M, Boccardi V, Vestini F, Lettieri $\mathrm{B}$, et al. Relationships between daily acute glucose fluctuations and cognitive performance among aged type 2 diabetic patients. Diabetes Care. 2010;33(10):2169-74.

30. Wei F, Sun X, Zhao Y, Zhang H, Diao Y, Liu Z. Excessive visit-tovisit glycemic variability independently deteriorates the progression of endothelial and renal dysfunction in patients with type 2 diabetes mellitus. BMC Nephrol. 2016;17(1):67.

31. Benjamin EM. Self-monitoring of blood glucose: the basics. Clin Diabetes. 2002;20:45-7.

32. Speight J, Browne JL, Furler JS. Testing times! Choosing wisely when it comes to monitoring type 2 diabetes. Med J Aust. 2015;203(9):354-6.

33. FDA. Blood glucose monitoring test systems for prescription point-of-care use. FDA Document issued on: October 11, 2016. 
Available at: https://www.fda.gov/downloads/medicaldevices/ deviceregulationandguidance/guidanced ocuments/ucm380325. pdf. Accessed on: April $3^{\text {rd }}, 2018$.

34. Clar C, Barnard K, Cummins E, Royle P, Waugh N, Aberdeen Health Technology Assessment Group. Self-monitoring of blood glucose in type 2 diabetes: systematic review. Health Technol Assess. 2010;14(12):1-140.

35. Parkin CG, Buskirk A, Hinnen DA, Axel-Schweitzer M. Results that matter: structured vs. unstructured selfmonitoring of blood glucose in type 2 diabetes. Diabetes Res Clin Pract. 2012;97(1):6-15.

36. Polonsky WH, Fisher L, Schikman CH, Hinnen DA, Parkin CG, Jelsovsky Z, et al. Structured self-monitoring of blood glucose significantly reduces A $1 \mathrm{C}$ levels in poorly controlled, noninsulin-treated type 2 diabetes: results from the structured testing program study. Diabetes Care. 2011;34(2):262-7.

37. Parkin CG, Hinnen DA, Tetrick DL. Effective use of structured self-management of blood glucose in type 2 diabetes: lessons from the STeP study. Clin Diabetes. 2011;29(4):131-8.

38. Klonoff DC, Blonde L, Cembrowski G, Chacra AR, Charpentier G, Colagiuri S, et al. Consensus report: the current role of selfmonitoring of blood glucose in non-insulin-treated type 2 diabetes. J Diabetes Sci Technol. 2011;5(6):1529-48.

39. Fisher L, Polonsky WH, Parkin CG, Jelsovsky Z, Petersen B, Wagner RS. The impact of structured blood glucose testing on attitudes toward self-management among poorly controlled, insulin-naïve patients with type 2 diabetes. Diabetes Res Clin Pract. 2012;96(2):149-55.

40. Bosi E, Scavini M, Ceriello A, Cucinotta D, Tiengo A, Marino R, et al. Intensive structured self-monitoring of blood glucose and glycemic control in noninsulin-treated type 2 diabetes: the PRISMA randomized trial. Diabetes Care. 2013;36(10):2887-94.

41. Czupryniak L, Barkai L, Bolgarska S, Bronisz A, Broz J, Cypryk $\mathrm{K}$, et al. Self-monitoring of blood glucose in diabetes: from evidence to clinical reality in central and Eastern Europe-recommendations from the international central-eastern European expert group. Diabetes Technol Ther. 2014;16(7):460-75.

42. Silva DD, Bosco AA. An educational program for insulin selfadjustment associated with structured self-monitoring of blood glucose significantly improves glycemic control in patients with type 2 diabetes mellitus after 12 weeks: a randomized, controlled pilot study. Diabetol Metab Syndr. 2015;7:2.

43. Klonoff DC. New evidence demonstrates that self-monitoring of blood glucose does not improve outcomes in type 2 diabetes-when this practice is not applied properly. J Diabetes Sci Technol. 2008;2(3):342-8.

44. Bonora E, Tuomilehto J. The pros and cons of diagnosing diabetes with A1C. Diabetes Care. 2011;34(Suppl 2):S184-90.

45. American Diabetes Association. 13. Management of diabetes in pregnancy: standards of medical care in diabetes 2018. Diabetes Care. 2018;41(Suppl 1):S137-43.

46. Karter AJ, Ackerson LM, Darbinian JA, D'Agostino RB Jr, Ferrara A, Liu J, et al. Self-monitoring of blood glucose levels and glycemic control: the Northern California Kaiser Permanente Diabetes registry. Am J Med. 2001;111(1):1-9.

47. Martin S, Schneider B, Heinemann L, Lodwig V, Kurth HJ, Kolb $\mathrm{H}$, et al. Self-monitoring of blood glucose in type 2 diabetes and long-term outcome: an epidemiological cohort study. Diabetologia. 2006;49(2):271-8.

48. Kesavadev J, Shankar A, Pillai PB, Krishnan G, Jothydev S. Costeffective use of telemedicine and self-monitoring of blood glucose via Diabetes Tele Management System (DTMS) to achieve target glycosylated hemoglobin values without serious symptomatic hypoglycemia in 1,000 subjects with type 2 diabetes mellitus-a retrospective study. Diabetes Technol Ther. 2012;14(9):772-6.
49. Klonoff DC. Benefits and limitations of self-monitoring of blood glucose. J Diabetes Sci Technol. 2007;1(1):130-2.

50. Shiraiwa T, Takahara M, Kaneto H, Miyatsuka T, Yamamoto K, Yoshiuchi K, et al. Efficacy of occasional self-monitoring of postprandial blood glucose levels in type 2 diabetic patients without insulin therapy. Diabetes Res Clin Pract. 2010;90(3):e91-2.

51. Ong WM, Chua SS, Ng CJ. Barriers and facilitators to selfmonitoring of blood glucose in people with type 2 diabetes using insulin: a qualitative study. Patient Prefer Adherence. 2014;8:237-46.

52. Ward JE, Stetson BA, Mokshagundam SP. Patient perspectives on self-monitoring of blood glucose: perceived recommendations, behaviors and barriers in a clinic sample of adults with type 2 diabetes. J Diabetes Metab Disord. 2015;14:43.

53. Simon J, Gray A, Clarke P, Wade A, Neil A, Farmer A, et al. Cost effectiveness of self monitoring of blood glucose in patients with non-insulin treated type 2 diabetes: economic evaluation of data from the DiGEM trial. BMJ. 2008;336(7654):1177-80.

54. O'Kane MJ, Bunting B, Copeland M, Coates VE, ESMON Study Group. Efficacy of self monitoring of blood glucose in patients with newly diagnosed type 2 diabetes (ESMON study): randomised controlled trial. BMJ. 2008;336(7654):1174-7.

55. Heisler M, Vijan S, Anderson RM, Ubel PA, Bernstein SJ, Hofer TP. When do patients and their physicians agree on diabetes treatment goals and strategies, and what difference does it make? J Gen Intern Med. 2003;18(11):893-902.

56. ISO In vitro diagnostic test systems-Requirements for bloodglucose monitoring systems for self-testing in managing diabetes mellitus. ISO 15197:2013. Available at: https://www.iso.org/ standard/54976.html Accessed on: $9^{\text {th }}$ March 2018.

57. Freckmann G, Schmid C, Baumstark A, Pleus S, Link M, Haug C. System accuracy evaluation of 43 blood glucose monitoring systems for self-monitoring of blood glucose according to DIN EN ISO 15197. J Diabetes Sci Technol. 2012;6(5):1060-75.

58. Baumstark A, Jendrike N, Liebing C, Haug C, Freckmann G. System accuracy and user performance evaluation of an improved system for self-monitoring of blood glucose. J Diabetes Sci Technol. 2018;12(2):407-11.

59. Tonyushkina K, Nichols JH. Glucose meters: a review of technical challenges to obtaining accurate results. J Diabetes Sci Technol. 2009;3(4):971-80.

60. Stephan P, Schmid C, Freckmann G, Pleus S, Haug C, Müller P. The rectangle target plot: a new approach to the graphical presentation of accuracy of systems for self-monitoring of blood glucose. J Diabetes Sci Technol. 2015;10(2):343-9.

61. Müller P, Hattemer A, Stephan P. Assessing system accuracy of blood glucose monitoring systems using rectangle target plots. J Diabetes Sci Technol. 2015;10(2):350-65.

62. Agborsangaya CB, Robitaille C, Dunbar P, Langlois MF, Leiter LA, Dai S, et al. Self-monitoring of blood glucose in type 2 diabetes: results of the 2011 survey on living with chronic diseases in Canada. Health Rep. 2013;24(6):3-8.

63. Barnard KD, Young AJ, Waugh NR. Self monitoring of blood glucose - a survey of diabetes UK members with type 2 diabetes who use SMBG. BMC Res Notes. 2010;3:318.

64. Barnett AH, Krentz AJ, Strojek K, Sieradzki J, Azizi F, Embong $\mathrm{M}$, et al. The efficacy of self-monitoring of blood glucose in the management of patients with type 2 diabetes treated with a gliclazide modified release-based regimen. A multicentre, randomized, parallel-group, 6-month evaluation (DINAMIC 1 study). Diabetes Obes Metab. 2008;10(12):1239-47.

65. Karter AJ, Parker MM, Moffet HH, Spence MM, Chan J, Ettner SL, et al. Longitudinal study of new and prevalent use of selfmonitoring of blood glucose. Diabetes Care. 2006;29(8):1757-63.

66. McAndrew L, Schneider SH, Burns E, Leventhal H. Does patient blood glucose monitoring improve diabetes control? A systematic 
review of the literature. Diabetes Educ. 2007;33(6):991-1011. discussion 1012-3

67. Miller KM, Beck RW, Bergenstal RM, Goland RS, Haller MJ, McGill JB, et al. Evidence of a strong association between frequency of self-monitoring of blood glucose and hemoglobin A1c levels in T1D exchange clinic registry participants. Diabetes Care. 2013;36(7):2009-14.

68. Morgan CL, Griffin A, Chamberlain GH, Turkiendorf A, McEwan P, Evans LM, et al. A longitudinal study into the new and long-term use of self-monitoring blood glucose strips in the UK. Diabetes Ther. 2010;1(1):1-9.

69. Schwedes U, Siebolds M, Mertes G, SMBG Study Group. Mealrelated structured self-monitoring of blood glucose: effect on diabetes control in non-insulin-treated type 2 diabetic patients. Diabetes Care. 2002;25(11):1928-32.

70. Ziegler R, Heidtmann B, Hilgard D, Hofer S, Rosenbauer J, Holl $\mathrm{R}$, et al. Frequency of SMBG correlates with HbAlc and acute complications in children and adolescents with type 1 diabetes. Pediatr Diabetes. 2011;12(1):11-7.

71. Diabetes Control and Complications Trial Research Group, Nathan DM, Genuth S, Lachin J, Cleary P, Crofford O, et al. The effect of intensive treatment of diabetes on the development and progression of long-term complications in insulin-dependent diabetes mellitus. N Engl J Med. 1993;329(14):977-86.

72. International Diabetes Federation Guideline Development Group. Global guideline for type 2 diabetes. Diabetes Res Clin Pract. 2014;104(1):1-52.

73. Bailey TS, Grunberger G, Bode BW, Handelsman Y, Hirsch IB, Jovanovič L, et al. American Association of Clinical Endocrinologists and American College of endocrinology 2016 outpatient glucose monitoring consensus statement. Endocr Pract. 2016;22(2):231-61.

74. Ji L, Su Q, Feng B, Shan Z, Hu R, Xing X, et al. Structured selfmonitoring of blood glucose regimens improve glycemic control in poorly controlled Chinese patients on insulin therapy: results from COMPASS. J Diabetes. 2017;9(5):495-501.

75. Ceriello A. Self-monitoring of blood glucose in type 2 diabetes: is the debate (finally) ending? Diabetes Res Clin Pract. 2012;97(1): $1-2$.

76. Davidson MB. The dilemma of self-monitoring of blood glucose. Diabetologia. 2007;50(3):497-9.

77. Welschen LM, Bloemendal E, Nijpels G, Dekker JM, Heine RJ, Stalman WA, et al. Self-monitoring of blood glucose in patients with type 2 diabetes who are not using insulin: a systematic review. Diabetes Care. 2005;28(6):1510-7.

78. Towfigh A, Romanova M, Weinreb JE, Munjas B, Suttorp MJ, Zhou A, et al. Self-monitoring of blood glucose levels in patients with type 2 diabetes mellitus not taking insulin: a meta-analysis. Am J Manag Care. 2008;14(7):468-75.

79. Malanda UL, Welschen LM, Riphagen II, Dekker JM, Nijpels G, Bot SD. Self-monitoring of blood glucose in patients with type 2 diabetes mellitus who are not using insulin. Cochrane Database Syst Rev. 2012;1:CD005060.

80. Farmer AJ, Perera R, Ward A, Heneghan C, Oke J, Barnett AH, et al. Meta-analysis of individual patient data in randomised trials of self monitoring of blood glucose in people with non-insulin treated type 2 diabetes. BMJ. 2012;e486:344.

81. Schnell O, Alawi H, Battelino T, Ceriello A, Diem P, Felton AM, et al. Self-monitoring of blood glucose in type 2 diabetes: recent studies. J Diabetes Sci Technol. 2013;7(2):478-88.

82. Parsons S, Luzio S, Owens DR. The SMBG study: structured selfmonitoring of blood glucose in non-insulin treated type 2 diabetes. IDF congress 2017; 4-8 December Abu Dhabi; Abstract OP0073.
83. Peel E, Douglas M, Lawton J. Self monitoring of blood glucose in type 2 diabetes: longitudinal qualitative study of patients' perspectives. BMJ. 2007;335(7618):493.

84. Fisher L, Polonsky W, Parkin CG, Jelsovsky Z, Amstutz L, Wagner RS. The impact of blood glucose monitoring on depression and distress in insulin-naïve patients with type 2 diabetes. Curr Med Res Opin. 2011;27(Suppl 3):39-46.

85. Allemann S, Houriet C, Diem P, Stettler C. Self-monitoring of blood glucose in non-insulin treated patients with type 2 diabetes: a systematic review and meta-analysis. Curr Med Res Opin. 2009;25(12):2903-13.

86. Durán A, Martín P, Runkle I, Pérez N, Abad R, Fernández M, et al. Benefits of self-monitoring blood glucose in the management of new-onset type 2 diabetes mellitus: the St Carlos study, a prospective randomized clinic-based interventional study with parallel groups. J Diabetes. 2010;2(3):203-11.

87. Franciosi M, Lucisano G, Pellegrini F, Cantarello A, Consoli A, Cucco L, et al. ROSES: role of self-monitoring of blood glucose and intensive education in patients with type 2 diabetes not receiving insulin. A pilot randomized clinical trial. Diabet Med. 2011:28(7):789-96.

88. Kempf K, Tankova T, Martin S. ROSSO-in-praxi-international: long-term effects of self-monitoring of blood glucose on glucometabolic control in patients with type 2 diabetes mellitus not treated with insulin. Diabetes Technol Ther. 2013;15(1):89-96.

89. Harashima S, Nishimura A, Ikeda K, Wang Y, Liu Y, Inagaki N. Once daily self-monitoring of blood glucose (SMBG) improves glycemic control in oral hypoglycemic agents (OHA)-treated diabetes: SMBG-OHA follow-up study. J Diabetes Sci Technol. 2015;10(2):378-82.

90. Farmer A, Wade A, Goyder E, Yudkin P, French D, Craven A, et al. Impact of self monitoring of blood glucose in the management of patients with non-insulin treated diabetes: open parallel group randomised trial. BMJ. 2007;335(7611):132.

91. Zarkogianni K, Litsa E, Mitsis K, Wu PY, Kaddi CD, Cheng $\mathrm{CW}$, et al. A review of emerging technologies for the management of diabetes mellitus. IEEE Trans Biomed Eng. 2015;62(12):2735-49.

92. DiaTribe (2015, April 16). Google secures patent for glucosesensing contact lens. Available at: Retrieved from https:// diatribe.org/google-secures-patent-glucose-sensing-contact-lens. Accessed on: $8^{\text {th }}$ March 2018.

93. Bajaj S. RSSDI clinical practice recommendations for the management of type 2 diabetes mellitus 2017. Int J Diabetes Dev Ctries. 2018;38(Suppl 1):1-115.

94. HAPO Study Cooperative Research Group, Metzger BE, Lowe LP, Dyer AR, Trimble ER, Chaovarindr U, et al. Hyperglycemia and adverse pregnancy outcomes. N Engl J Med. 2008;358(19): 1991-2002.

95. Crowther CA, Hiller JE, Moss JR, McPhee AJ, Jeffries WS, Robinson JS, et al. Effect of treatment of gestational diabetes mellitus on pregnancy outcomes. N Engl J Med. 2005;352(24): 2477-86.

96. IDF Clinical Guidelines Task Force. Global guideline on pregnancy and diabetes. Brussels: International Diabetes Federation; 2009.

97. Wahabi HA, Alzeidan RA, Esmaeil SA. Pre-pregnancy care for women with pre-gestational diabetes mellitus: a systematic review and meta-analysis. BMC Public Health. 2012;12:792.

98. Negrato CA, Zajdenverg L. Self-monitoring of blood glucose during pregnancy: indications and limitations. Diabetol Metab Syndr. 2012;4(1):54.

99. NHM Diagnosis \& management of gestational diabetes mellitus, technical and operational guidelines, by Maternal Health Division Ministry of Health and Family Welfare, Government of India, February 2018. Available at: http://nhm.gov.in/New_Updates 
2018/NHM_Components/RMNCH_MH_Guidelines/Ge stational-Diabetes-Mellitus.pdf. Accessed on: $1^{\text {st }}$ May 2018.

100. Gupta S, Gupta K, Gathe S, Bamhra P, Gupta S. Insulin therapy in women with pregestational type 2 diabetes and its relevance to maternal and neonatal complications. Int J Diabetes Dev Ctries. 2018;38:47. https://doi.org/10.1007/s13410-016-0541-2.

101. Schnell O, Alawi H, Battelino T, Ceriello A, Diem P, Felton A, et al. Addressing schemes of self-monitoring of blood glucose in type 2 diabetes: a European perspective and expert recommendation. Diabetes Technol Ther. 2011;13(9):959-65.

102. International Diabetes Federation. IDF guideline on selfmonitoring of blood glucose in non-insulin treated type 2 diabetes. Brussels, 2009. Available at: https:/www.idf.org/e-library/ guidelines/85-self-monitoring-of-blood-glucose-in-non-insulintreated-type-2-diabetes.html Accessed on $29^{\text {th }}$ December 2017.

103. Unnikrishnan R, Mohan V. Protocols of SMBG in various insulin regimens - a practical guide for titration and control. Frontiers in Medicine. 2009;23:39-41.

104. SMBG International Working Group. Self-monitoring of blood glucose in type 2 diabetes: an inter-country comparison. Diabetes Res Clin Pract. 2008;82(3):e15-8.
105. Nagpal J, Bhartia A. Quality of diabetes care in the middle- and high-income group populace: the Delhi diabetes community (DEDICOM) survey. Diabetes Care. 2006;29(11):2341-8.

106. Davis WA, Bruce DG, Davis TM. Is self-monitoring of blood glucose appropriate for all type 2 diabetic patients? The fremantle diabetes study. Diabetes Care. 2006;29(8):1764-70.

107. Mastura I, Mimi O, Piterman L, Teng CL, Wijesinha S. Selfmonitoring of blood glucose among diabetes patients attending government health clinics. Med J Malaysia. 2007;62(2):147-51.

108. Mohan V, Deepa R, Shefali AK, Poongothai S, Monica M, Karkuzhali K. Evaluation of one touch HORIZON—a highly affordable glucose monitor. J Assoc Physicians India. 2004;52:779-82.

109. Krishnan V, Thirunavukkarasu J. Assessment of knowledge of self blood glucose monitoring and extent of self titration of anti-diabetic drugs among diabetes mellitus patients - a cross sectional, community based study. J Clin Diagn Res. 2016;10(3):FC09-11.

110. Kirkman MS, Briscoe VJ, Clark N, Florez H, Haas LB, Halter JB, et al. Diabetes in older adults: a consensus report. J Am Geriatr Soc. 2012;60(12):2342-56.

\section{Affiliations}

P. V. Rao ${ }^{1}$ - B. M. Makkar ${ }^{2} \cdot$ Ajay Kumar $^{3}$ - A. K. Das ${ }^{4}$ A. K. Singh ${ }^{5} \cdot$ Ambrish Mithal $^{6} \cdot$ Anil Bhansali $^{7} \cdot$ Anoop Misra $^{8,9}$.

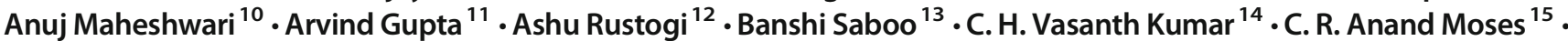
Hemant Thacker ${ }^{16}$. Jayant Panda ${ }^{17}$. Jayaprakashsai Jana ${ }^{14}$. Jothydev Kesavdev ${ }^{18} \cdot$ K. R. Narasimha Setty ${ }^{19}$. Manoj Chawla ${ }^{20}$ - Neeta Deshpande ${ }^{21}$ - Nikhil Tandon ${ }^{22}$ - Rajeev Chawla ${ }^{23}$. Rajeev Kovil ${ }^{24}$. Rakesh Sahay ${ }^{25}$. S. V. Madhu ${ }^{26}$. Samar Banerjee ${ }^{27}$. Sanjay Agarwal ${ }^{28}$. Sanjay Kalra ${ }^{29}$. Sarita Bajaj ${ }^{30}$. Shashank R Joshi ${ }^{31}$.

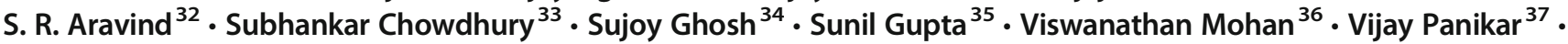
Vijay Viswanathan ${ }^{38}$

1 Kumudini Devi Diabetes Research Center, Ramdevrao Hospital, Hyderabad, India

2 Dr. Makkar's Diabetes and Obesity Centre, Delhi, India

3 Diabetes Care and Research Centre, Patna, India

4 Department of Endocrinology, Pondicherry Institute of Medical Sciences, Pondicherry, India

$5 \quad$ G.D Hospital and Diabetes Institute, Kolkata, India

6 Medanta Medicity, Gurgaon, India

7 Department of Endocrinology, PGIMER, Chandigarh, India

8 Fortis-CDOC Center of Excellence for Diabetes, Metabolic Diseases, and Endocrinology, New Delhi, India

9 National Diabetes, Obesity and Cholesterol Foundation and Diabetes Foundation, New Delhi, India

10 Department of Internal Medicine, BBD University, Lucknow, India

11 Jaipur Diabetes Research Centre, Jaipur, India

12 Department of Endocrinology and Metabolism, Post Graduate Institute of Medical Education and Research (PGIMER), Chandigarh, India

13 DIA Care - Diabetes Care and Hormone Clinic, Ahmedabad, India
14 Apollo Hospitals, Hyderabad, India

15 The Tamil Nadu Dr. MGR Medical University, Chennai, India

16 Jaslok, Breach Candy and Bhatia Hospital, Mumbai, India

17 Department of Medicine, SCB Medical College, Cuttack, India

18 Jyothydev's Diabetes Research Centres, Thiruvananthapuram, Kerala, India

19 Karnataka Institute of Diabetology, Bengaluru, India

20 Lina Diabetes Care Centre, Honorary Diabetologist, Asian Heart Institute \& S L Raheja Fortis, Mumbai, India

21 USM- KLE International Medical Programme; Belgaum Diabetes Centre, Belgaum, India

22 Department of Endocrinology and Metabolism, All India Institute of Medical Sciences, New Delhi 110029, India

23 North Delhi Diabetes Centre, Delhi, India

24 Dr. Kovils Diabetes Care Centres, Nanavati Hospital, S LRaheja Hospital Arogya, Nidhi Hospital, BSES Global Hospital, Mumbai, India

25 Department of Endocrinology, Osmania Medical College and General Hospital, Hyderabad, India 
26 Department of Medicine, University College of Medical Sciences, Delhi, India

27 Department of Medicine, Vivekananda institute of Medical Sciences, Kolkata, India

28 Aegle Clinic-Diabetes Care, Pune, India

29 Bharti Research Institute of Diabetes and Endocrinology (BRIDE), Chandigarh, Haryana, India

30 Department of Medicine, MLN Medical College, Allahabad, India

31 Joshi Hospital, Mumbai, India
32

33

Department of Endocrinology and Metabolism, PGME\&R and SSKM Hospital, Kolkata, India

34 AMRI Hospital, Kolkata, India

35 Sunil's Diabetes Care n' Research Centre Pvt. Ltd., Nagpur, India

36 Dr. Mohan's Diabetes Specialities Centre, Chennai, India

37 Dr. Panikar's Speciality Care Centre, Mumbai, India

38 MV Hospital for Diabetes, Chennai, India 